\title{
A IMPORTÂNCIA DO FUTEBOL EM ATIVIDADES EXTENSIONISTAS: UM RELATO DE EXPERIÊNCIA DO CERFUT/DEF/UEM
}

\author{
Matheus de Oliveira Jaime, Universidade Estadual de Maringá - UEM, Maringá, Paraná \\ - Brasil \\ Paulo Henrique Borges, Universidade Estadual de Maringá - UEM, Maringá, Paraná - \\ Brasil \\ Vanessa Menezes Menegassi, Universidade Estadual de Maringá - UEM, Maringá, \\ Paraná - Brasil \\ Edielson Frigeri Leite Ciqueira, Universidade Estadual de Maringá - UEM, Maringá, \\ Paraná - Brasil \\ Luan Antônio Corá Silveira, Universidade Estadual de Maringá - UEM, Maringá, Paraná \\ - Brasil \\ Marcos Aurélio de Oliveira Magossi, Universidade Estadual de Maringá - UEM, \\ Maringá, Paraná - Brasil \\ Wilson Rinaldi, Universidade Estadual de Maringá - UEM, Maringá, Paraná - Brasil
}

\section{RESUMO}

Este presente artigo é um relato de experiência de acadêmicos da Universidade Estadual de Maringá (UEM), baseado em vivências no Centro Regional de Formação em Futebol (CERFUT), com o objetivo de fazer um apanhado teórico-conceitual e prático, sobre a importância da maximização de atividades extensionistas que almejam a interação entre conhecimento científico e intervenções práticas e que tenham como visão a formação integral de jovens jogadores de futebol e profissionais de Educação Física.

Palavras-Chave: Atividades extensionistas; Educação física; Futebol; Formação integral.

\section{THE SOCCER IMPORTANCE IN EXTENSIONISTS ACTIVITIES: A REPORT OF EXPERIENCE CERFUT/DEF/UEM}

\begin{abstract}
This present article is a report of academic experience at the State University of Maringá (UEM), based on experiences in the Regional Training Centre in Football (CERFUT), in order to make a theoretical-conceptual and practical overview on the importance of maximizing extension activities that aims the interaction between scientific knowledge and practical interventions and which have as vision the integral formation of young football players and physical education professionals.
\end{abstract}

Key-Words: Extensionists activities; Physical education; Soccer; Integral formation.

Conexões: revista da Faculdade de Educação Física da UNICAMP, Campinas, v. 13, n. 4, p. 155-164, out./dez. 2015. ISSN: 1983-9030. 


\section{LA IMPORTANCIA DEL FÚTBOL EN ACTIVIDADES DE EXTENSIÓN: UN INFORME DE EXPERIENCIA DEL CERFUT/DEF/UEM}

\section{RESUMEN}

El presente artículo esun informe de laexperiencia académica enlaUniversidad Estatal de Maringá (UEM) basadoenexperienciasenel Centro Regional de FormaciónenFútbol (CERFUT), conelfin de hacer una descripción general teórico-conceptual y práctico sobre laimportancia de maximización de lasactividades de extensión que tienen como objetivo lainteracción entre elconocimiento científico y las intervenciones prácticasy cuyavisiónsea de la formación integral de losjóvenesjugadores de fútbol y losprofesionales de educación física.

Palabras-Clave: Actividades extensionistas; Educación física; Fútbol; Formación integral. 


\section{INTRODUÇÃO}

No país onde o futebol é considerado patrimônio cultural, suas discussões ocupam um espaço privilegiado especialmente neste momento de pós realização do maior evento deste esporte, a Copa do Mundo da FIFA. Após a Seleção Brasileira sucumbir perante a campeã Alemanha, levantou-se a questão da necessidade de melhorar a formação de crianças e adolescentes praticantes da modalidade não somente para o esporte, mas sim de maneira íntegra pelo esporte, uma vez que o esporte é considerado uma forma de sociabilização e de transmissão de valores. ${ }^{1}$

Nesse sentido, os benefícios do esporte têm ultrapassado o limite do bem estar físico e tornam-se visíveis também a nível educacional e formativo para crianças e adolescentes. ${ }^{2}$ Requer do futebolista o desempenho ótimo de capacidades biomotoras como resistência aeróbia, potência anaeróbia, velocidade de deslocamento, agilidade e força explosiva. ${ }^{3}$ Para tal, se torna necessário que esses jogadores mantenham ou adquiram algumas características relacionadas à composição corporal como peso corporal, massa magra e percentual de gordura corporal, em níveis ideais ou compatíveis com os níveis de um atleta da modalidade e da categoria, visto que de acordo com Mantovani et al., ${ }^{4}$ índices elevados da gordura corporal estão associados com o mal condicionamento físico e queda de rendimento na maioria das modalidades. $\mathrm{AACSM}^{5}$ define a capacidade cardiorrespiratória como componente da aptidão física e, treinar esta capacidade pode ajudar a manter e melhorar vários aspectos da função cardiovascular que podem resultar na melhora da qualidade de vida.

Conseqüentemente, devido ao grande apelo junto às populações jovens e à comunidade, somado ao fato de ser um esporte onde os praticantes permanecem por muito tempo na modalidade, a hegemonia do futebol como prática esportiva mundial se faz notória. ${ }^{6} \mathrm{O}$ desenvolvimento de políticas esportivas facilitadoras e fomentadoras para a proposição de projetos e programas esportivos faz todo o sentido, uma vez que estes projetos contribuem diretamente da melhoria de vida da população jovem. As universidades, cujo objetivo é estimular a pesquisa, o ensino e a extensão, devem participar por meio da proposição de

Conexões: revista da Faculdade de Educação Física da UNICAMP, Campinas, v. 13, n. 4, p. 155-164, out./dez. 2015. ISSN: 1983-9030. 
projetos de extensão contemplando interativamente o ensino e a pesquisa num ambiente complexo e profícuo de retorno do conhecimento à comunidade.

Assim, o objetivo deste estudo é relatar as experiências obtidas com um projeto de extensão de futebol na Universidade Estadual de Maringá (UEM), buscando compreender a importância da criação deste tipo de projeto sobre a vida de crianças e adolescentes e sobre a comunidade científica.

\section{CERFUT}

O projeto de extensão CERFUT, denominado Centro Regional de Formação em Futebol, foi criado em 2010 na UEM tendo o futebol como meio de contribuição para formação de crianças, adolescentes e jovens, já que este esporte tem sido nos últimos cinqüenta anos uma atividade de grande importância social cujas conseqüências transcendem as linhas do campo de jogo, tornando-se mesmo questões de Estado. ${ }^{7}$

Segundo Stephanou et al. ${ }^{8}$ [...] os projetos sociais nascem do desejo de mudar uma realidade. Os projetos são pontes entre o desejo e a realidade. São ações estruturantes e intencionais, de um grupo ou organização social, que partem da reflexão e do diagnóstico sobre determinada problemática e buscam contribuir, em alguma medida, para outro mundo possível.

O projeto visa ser proativo diante dos alunos matriculados e entende que o futebol na sua expressão multitudinária não é apenas um espetáculo desportivo, mas também um meio de educação física/desportiva e um campo de aplicação da ciência. ${ }^{9-10}$

Por conseguinte, os objetivos do projeto são amplos e giram em torno de oportunizar a prática do futebol às crianças e adolescentes de Maringá e região organizando uma estrutura temporal de formação considerando as fases de estimulação, aprendizagem, prática e aperfeiçoamento, especialização, treinamento e manutenção na modalidade de futebol. ${ }^{11}$ Além de orientar o desenvolvimento geral dos praticantes de futebol a partir de uma concepção complexa de ser, sociedade e mundo, o projeto também contempla o aprimoramento dos aspectos técnicos, táticos e físicos, possibilitando a formação de 
futuros jogadores para a modalidade. Evidências científicas acrescentam que a formação de atletas de elite geralmente se inicia em ambientes propícios à experimentação regular de atividades esportivas nos primeiros anos de vida. ${ }^{12}$

\section{ESTRUTURA FÍSICA E HUMANA}

O CERFUT está situado no Departamento de Educação Física (DEF) da UEM, onde possui 2 campos para realização de treinamentos. Desde a sua formação, o projeto contou com a participação de quinze acadêmicos de Educação Física, cinco graduados, um mestre e dois doutores, que atenderam e atendem as categorias: Sub-9, Sub-11, Sub-13, Sub-15 e Sub17. Estima-se que o CERFUT já atendeu aproximadamente 800 crianças e adolescentes desde a sua fundação.

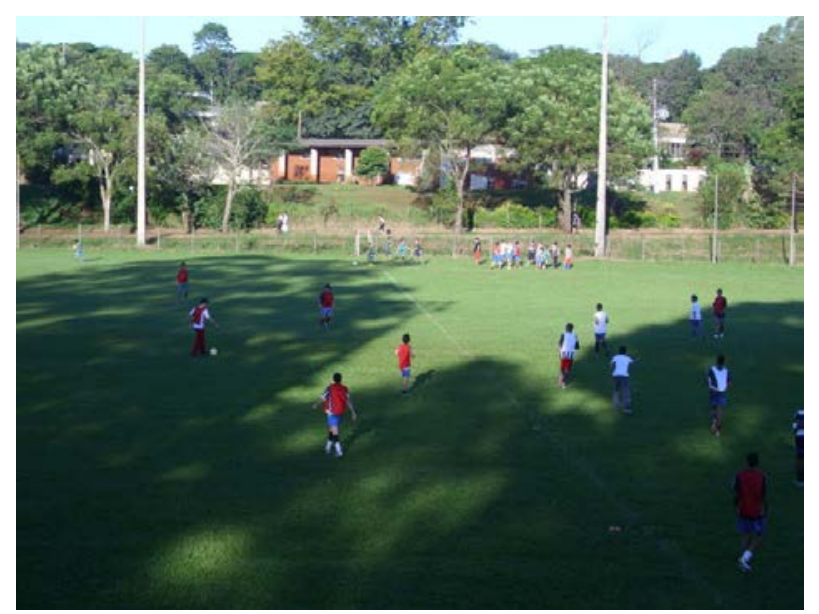

Figura 1: Treinamentos no campo de futebol do DEF.

As categorias são fracionadas de acordo com a idade e trabalhadas de maneira apropriada para cada idade específica, pois reconhecemos a importância em adaptar os materiais, espaços físicos e algumas regras, tendo em vista que existem diferenças estruturais, cognitivas, motoras e afetivas entre um adulto, um adolescente e uma criança na participação em atividades esportivas. ${ }^{13}$ As categorias sub-13, sub-15 e sub-17 participam de campeonatos regionais, enquanto as categorias sub-11 e sub-9 jogam torneios de curta duração onde o tamanho do campo e as exigências competitivas são menores.

Atualmente, ao entrar nas categorias do projeto, os alunos são avaliados por acadêmicos integrantes do CERFUT. São realizados vários testes antropométricos (massa corporal, 
estatura, altura tronco-cefálica, percentual de gordura e circunferências), ${ }^{14}$ maturacionais (pico de velocidade de crescimento) ${ }^{15}$, físicos (preensão manual, saltos verticais, resistência aeróbica, resistência muscular abdominal, velocidade $30 \mathrm{~m})^{16-17}$ e táticos ${ }^{18}$ com protocolos validados e amplamente utilizados no campo da Educação Física.

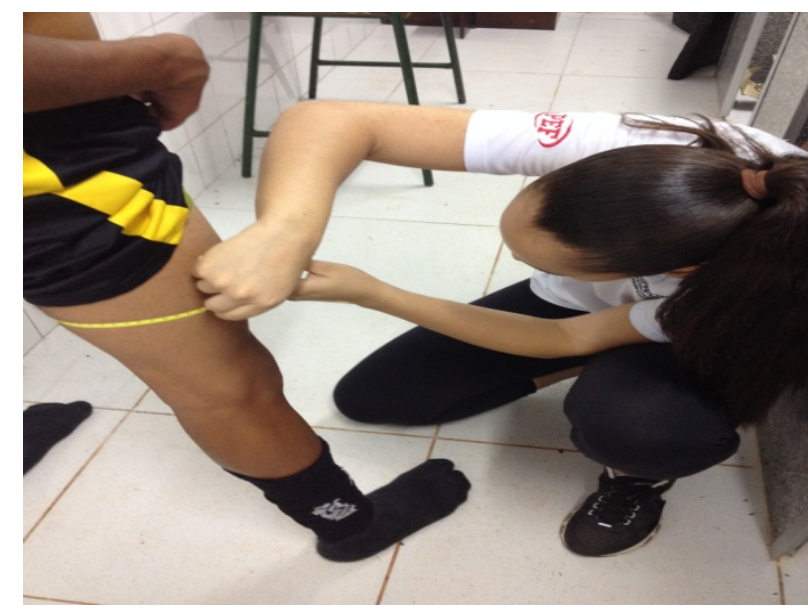

Figura 2: Avaliação antropométrica dos jogadores do CERFUT.

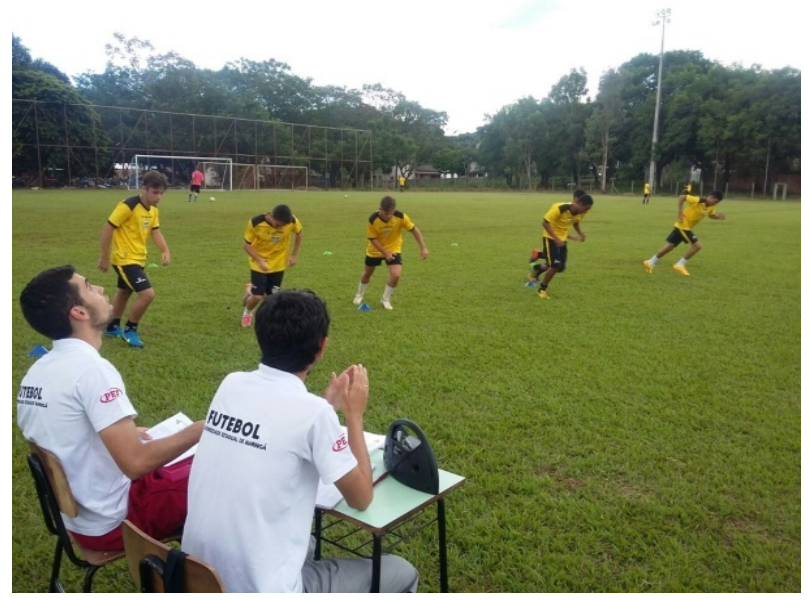

Figura 3: Avaliação física dos jogadores do CERFUT.

As informações provenientes dos testes são repassadas para os treinadores de cada categoria para que possam utilizar no planejamento das sessões de treinamento.

Conexões: revista da Faculdade de Educação Física da UNICAMP, Campinas, v. 13, n. 4, p. 155-164, out./dez. 2015. ISSN: 1983-9030. 


\section{CONQUISTAS}

Embora os resultados dos jogos sejam secundários para o cumprimento dos objetivos do projeto, algumas participações em campeonatos foram destacadas, como o vicecampeonato da Liga Desportiva de Maringá no ano de 2011 e o vice-campeonato na $1^{\circ}$ Copa Altônia do Mercosul em 2012. A participação da equipe sub-15 em 2011 estimulou seu treinador e acadêmico do curso de Educação Física a escrever um livro sobre os comportamentos táticos defensivos no futebol relacionados ao sistema 1-4-4-2 em losango, sistema este utilizado na referida equipe.

A interação entre conhecimento científico e as intervenções dos membros do projeto sempre foi uma marca do CERFUT. Nos seus primeiros cinco anos de existência (de 2010 a 2015), os acadêmicos participantes apresentaram resumos em oito congressos internacionais ou nacionais, publicaram quatro artigos científicos em periódicos recomendados pela CAPES e realizaram quatro trabalhos de conclusão de curso. Três acadêmicos já participaram do Programa Ciência sem Fronteiras, onde foram estudar futebol e a Educação Física em centros de excelência na Europa. Atualmente, existe uma dissertação de mestrado em andamento relacionada ao projeto, além de dois projetos de iniciação científica.

As pesquisas, a participação em congressos e os intercâmbios são fundamentais para qualificar as ações e intervenções dos membros e acadêmicos do curso, permitindo tanto melhorar os treinos quanto complementar a formação desses profissionais. Neste sentido, torna-se importante um staff composto por membros qualificados e alinhados com o propósito de entender e contribuir para o desenvolvimento esportivo integral de jovens jogadores sem ter pressa em especializá-los precocemente, demonstrando mais uma preocupação com uma formação plural e multilateral. ${ }^{19}$

\section{CONCLUSÃO}

Este relato demonstrou a viabilidade e a importância do projeto CERFUT na vida de crianças, adolescentes e acadêmicos de Educação Física. Ao longo dos anos, várias dificuldades foram vividas por aqueles que atuam diretamente no projeto, tais como falta de materiais de consumo e dificuldade com o transporte para os jogos, por exemplo. No Conexões: revista da Faculdade de Educação Física da UNICAMP, Campinas, v. 13, n. 4, p. 155-164, out./dez. 2015. ISSN: 1983-9030. 
entanto, apesar das dificuldades impostas pelo conjunto de questões, prospectamos seguir maximizando a oferta da prática esportiva contribuindo com o suporte necessário na capacitação de aspectos desportivos e sociais, sem deixar de lado nosso compromisso com o desenvolvimento acadêmico e da área de Educação Física.

\section{REFERÊNCIAS}

${ }^{1}$ ALMEIDA, M. A. B.; GUTIERREZ, G. L. Esporte e sociedade. EFDeportes.com: revista digital. Buenos Aires, n. 133, p. 1-8, 2009. Disponível em: $<$ http://www.efdeportes.com/efd133/esporte-e-sociedade.htm>Acesso em: 09 jul. 2015.

${ }^{2}$ BASSANI, J. J.; TORRI, D.; VAZ, A. F. Sobre a presença do esporte na escola: paradoxos e ambiguidades. Movimento, Porto Alegre, v. 9, n. 2, p. 89-112, maio/ago. 2003.

${ }^{3}$ CASTAGNA, C. et al. Aerobic fitness and yo-yo continuous and intermittent tests performance in soccer players: a correlation study. Journal of Strength and Conditioning Research, Colorado Springs, v. 20, n. 2, p. 320-325, may 2006.

${ }^{4}$ MANTOVANI, T. V. L. et al. Composição corporal e limiar anaeróbio de jogadores de futebol das categorias de base. Revista Mackenzie de Educação Física e Esporte, São Paulo, v. 7, n. 1, p. 25-33, 2008.

${ }^{5}$ ROSCAMP, R; SANTOS, M. G. A qualidade de vida relacionada à aptidão física e saúde. EFDeportes.com: revista digital, Buenos Aires, ano 16, n. 161, out. 2011. Disponível em: $<$ http://www.efdeportes.com/efd161/a-qualidade-de-vida-e-saude.htm> Acesso em: 18 jul. 2015.

${ }^{6}$ LOBATO, R. S.; REIS, A. L. T. Um estudo sobre aspectos motivacionais em crianças de 7 a 14 anos na escolha do futebol como modalidade esportiva. EFDeportes.com: revista digital, Buenos Aires, ano 15, n. 154, mar. 2011. Disponível em: 
<http://www.efdeportes.com/efd154/aspectos-motivacionais-na-escolha-do-futebol.htm> Acesso em: 18 jul. 2015.

7GASTALDO, E. L. A nação e o anuncio: a representação do "brasileiro" na publicidade da copa do mundo. 2009. 353 f. Tese (Doutorado em Educação Física) - Faculdade de Educação Física, Universidade Estadual de Campinas, Campinas, 2009.

${ }^{8}$ STEPHANOU, L.; MULLER, L. H.; CARVALHO, I. C. M. Guia para a elaboração de projetos sociais. Porto Alegre: Sinodal e Fundação Luterana de Diaconia, 2003.

${ }^{9}$ GARGANTA, J. O treino da táctica e da técnica nos jogos desportivos à luz do compromisso cognição-acção. In: BARBANTI, J. V. et al Esporte e actividade física: interacção entre rendimento e saúde. São Paulo: Manole, 2002.

${ }^{10}$ GARGANTA， J. Competências no ensino e treino de jovens futebolistas. EFDeportes.com: revista digital, Buenos Aires, n. 45, v. 8, fev. 2002. Disponível em: < http://www.efdeportes.com/efd45/ensino.htm> Acesso em: 09 jul. 2015.

${ }^{11}$ OLIVEIRA, V.; PAES, R. R. A pedagogia da iniciação esportiva: um estudo sobre o ensino dos jogos desportivos coletivos. EFDeportes.com: revista digital, Buenos Aires, $\mathrm{n}$. 71, v. 10, abr. 2004. Disponível em: <http://www.efdeportes.com/efd71/jogos.htm> Acesso em: 17 jul. 2015.

${ }^{12}$ COSTA, I. T.; CARDOSO, F. S. L.; GARGANTA, J. O Índice de desenvolvimento humano e a data de nascimento podem condicionar a ascensão de jogadores de futebol ao alto nível de rendimento? Motriz: revista de educação física, Rio Claro, v .19, n.1, 2013.

${ }^{13}$ GRAÇA, A.; OLIVEIRA, J. O ensino dos jogos desportivos. 3. ed. Rainho \& Neves, Lisboa: Santa Maria da Feira, 1998.

Conexões: revista da Faculdade de Educação Física da UNICAMP, Campinas, v. 13, n. 4, p. 155-164, out./dez. 2015. ISSN: 1983-9030. 
${ }^{14}$ GUEDES, D. P.; GUEDES, J. E. R. P. Manual prático para avaliação em educação física. Barueri: Manole, 2006.

${ }^{15}$ MIRWALD, R, L. et al. An assessment of maturity from anthropometric measurements. Medicine \& Science in Sports \& Exercise, v. 34, n.4, p. 689-694, apr. 2002.

${ }^{16}$ KRUSTRUP, P. The yo-yo intermittent recovery test: physiological response, reliability, and validity. Medicine \& Science in Sports \& Exercise, v. 35, n. 4, p. 697-705, apr. 2003.

${ }^{17}$ BOSCO, C. A dynamometer for evaluation of dynamic muscle work. European Journal of Applied Physiology and Occupational Physiology, v. 70, n. 5, p. 379-386, sept. 1995.

${ }^{18}$ COSTA, I, T. Sistema de avaliação táctica no Futebol (FUT-SAT): desenvolvimento e validação preliminar. Motricidade, v. 7, n. 1, p. 69-84, 2011.

${ }^{19}$ BOMPA, T. O. Treinamento total para jovens campeões. Campinas: Manole, 2002.

Recebido em: 19 jul. 2015

Aceito em:16 out. 2015

Contato: pauloborges.uem@gmail.com

Conexões: revista da Faculdade de Educação Física da UNICAMP, Campinas, v. 13, n. 4, p. 155-164, out./dez. 2015. ISSN: 1983-9030. 